\title{
Case Report: Multiple Tuberculous Tenosynovitis of the Flexor Tendons of the Wrist and Hand
}

\author{
Jinjun Liang ${ }^{1}$, Dan Yang ${ }^{1}$, Xuhao Yang ${ }^{1}$, Yunjun $\mathrm{Wu}^{1}$, Guo $\mathrm{Fu}^{1}$
}

${ }^{1}$ Department of Spine and Orthopedic Trauma, The First Affiliated Hospital of Jinan University, Guangzhou, China

\begin{abstract}
Background: Tuberculosis is a significant public health challenge and tuberculous tenosynovitis is therefore a rare form of musculoskeletal tuberculosis which usually affects the flexor tendons of the hand and wrist. Case presentation: We present a case of a 63-year-old male. He presented multiple progressively enlarging mass over the volar side of the right hand and wrist. He did not have a previous history of tuberculosis. However, his erythrocyte sedimentation rate was high and tuberculosis antibody test was slightly positive. Radiograph of chest showing multiple nodular and patchy dense shadows in bilateral lungs, and localized soft tissue mass around the radiocarpal joint. Excision biopsy of the mass was done and samples sent for histopathology comment. The findings were an inflamed, thickened synovia: suggestive of tuberculous tenosynovitis. Anti-tuberculous chemotherapy was commenced on the second postoperative day. Conclusion: Tuberculous tenosynovitis of the wrist is rare. However, in developing area where tuberculosis is prevalent, it should be part of the differential diagnosis of compound palmar ganglion in order to prevent delayed diagnosis and treatment.
\end{abstract}

Keywords: Tenosynovitis, Wrist, Hand, Flexor Tendons, Mycobacterium Tuberculosis, Tuberculosis

\section{Introduction}

Tuberculous tenosynovitis is an infrequent lesion for hand surgeons.(1) It usually results from hematogenous spread from a primary source like lungs, spine, and lymph nodes. It may cause chronic non-specific symptoms and signs of the tendon and synovium.(2) The patients may present with slowly increasing pain in the wrist, swelling around the wrist or hand, and limited range of motion which are more easily to lead to delayed diagnosis.(3) Therefore, it is critical to reach a definite diagnosis through histopathology early, followed by thorough surgical excision of all the tissues affected. Moreover, the appropriate anti-tuberculous chemotherapy should be applied immediately once the pathological diagnosis is confirmed.(4) The aim of this case report was to present the case of a patient with no history of tuberculosis who required surgical treatment for multiple masses located on the palm and wrist of his right hand.

\section{Case Report}

A 63-year-old well-nourished male presented to our hospital with a 7-year history of five progressively enlarging masses over the volar side of the right wrist and thumb, middle finger and little finger. It was associated with gradually increasing pain around the wrist which initially responded to nonsteroidal anti-inflammatory drugs. A month ago, he presented to the local hospital and underwent a B-ultrasound exam which showed: "Multiple ganglion cysts may occur."

The patient also had slight stiffness at the wrist joint and paraesthesia involving the distribution of the ulna nerve (ulna one and half fingers). These symptoms occasionally woke her up at night. He was a farmer and had no history of trauma injury around the wrist or fingers, no contact with any patient with tuberculosis, and no symptoms suggestive of rheumatoid arthritis. The patient had no previous history of diabetes and had not been vaccinated against tuberculosis.

\begin{abstract}
Physical examination showed a mass of about $8 \mathrm{~cm} \times 4$ $\mathrm{cm}$ on the right wrist of the patient, with tough texture, smooth surface, poor range of motion, no tenderness, no skin rash and ulcer on the surface, and a $2 \times 3 \mathrm{~cm}$ mass on the proximal end of the right thumb and middle finger, which was tough, tenderness-free and difficult to clench (Figure 1). He felt slightly numb on the half of the ulnar side of the ring finger and the little finger while normal on the other fingers.
\end{abstract}

X-ray photos of chest showing multiple nodular and patchy dense shadows in bilateral lungs, and localized soft tissue mass around the radiocarpal joint (Figure 2 A, B, C). Ultrasound examination of the wrist and hand showed diffuse inflammation and local effusion of tendon sheath of superficial and deep flexor tendon of right wrist, and flex tendon sheath of right thumb, middle finger and little finger (Figure 2 D, E, F). CRP (82.32) and erythrocyte sedimentation rate (ESR) increased $(104 \mathrm{~mm} / \mathrm{h})$, and mycobacterium tuberculosis antibody test showed weak positive. However, procalcitonin 0.069 did not increase significantly, and no mycobacterium tuberculosis was found in sputum examination and throat swabs. 
During the operation, the mass on the wrist did not extend through the flexor retinaculum, the mass contained a small amount of yellowish fluid, and the flexor tendon sheath was significantly thickened (Figure 3). While exploring the carpal tunnel and the Guyon's canal, the ulnar nerve was slightly compressed, but the epineurium of ulna nerve was not invaded. The masses of thumb, middle finger and little finger were also confined to the tendon sheath. The resected mass was yellowish, dense and uniform, and no obvious rice body was found. All tumors underwent total tendon sheath resection, and the specimens were sent for histopathology and culture.

The Ziehl-Neelsen staining of this material did not show any acid resistant bacteria. On histopathologic examination, granulomatous inflammation with Langerhans giant cells were observed. Culture was negative for acid-fast bacilli (Figure 4 A, B, C, D). In terms of anti-tuberculosis chemotherapy, the first two months of anti-tuberculosis chemotherapy included isoniazid, ethambutol, pyrazinamide and rifampicin and isoniazid and rifampicin for another 4 months. There was no recurrence after follow-up of 2 years (Figure 4 E, F).

FIGURE 1 (A) and (B) Clinical picture before operation showing multiple palmar masses of right wrist and hand.

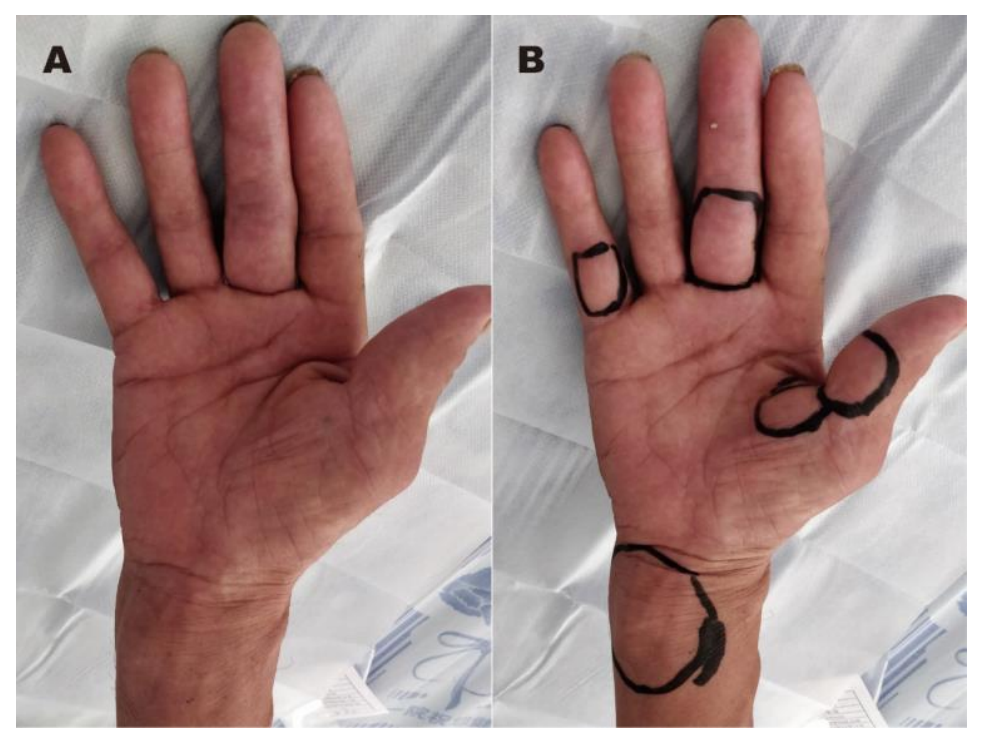

FIGURE 2 (A) X-ray of the chest showing multiple nodular and patchy dense shadows in bilateral lungs; (B) and (C) X-ray of the wrist showing localized soft tissue mass around the radiocarpal joint; (D), (E) and (F) Ultrasound examination showing diffuse inflammation and local effusion of tendon sheath of superficial and deep flexor tendon of right wrist, and flex tendon sheath of right thumb, middle finger and little finger.

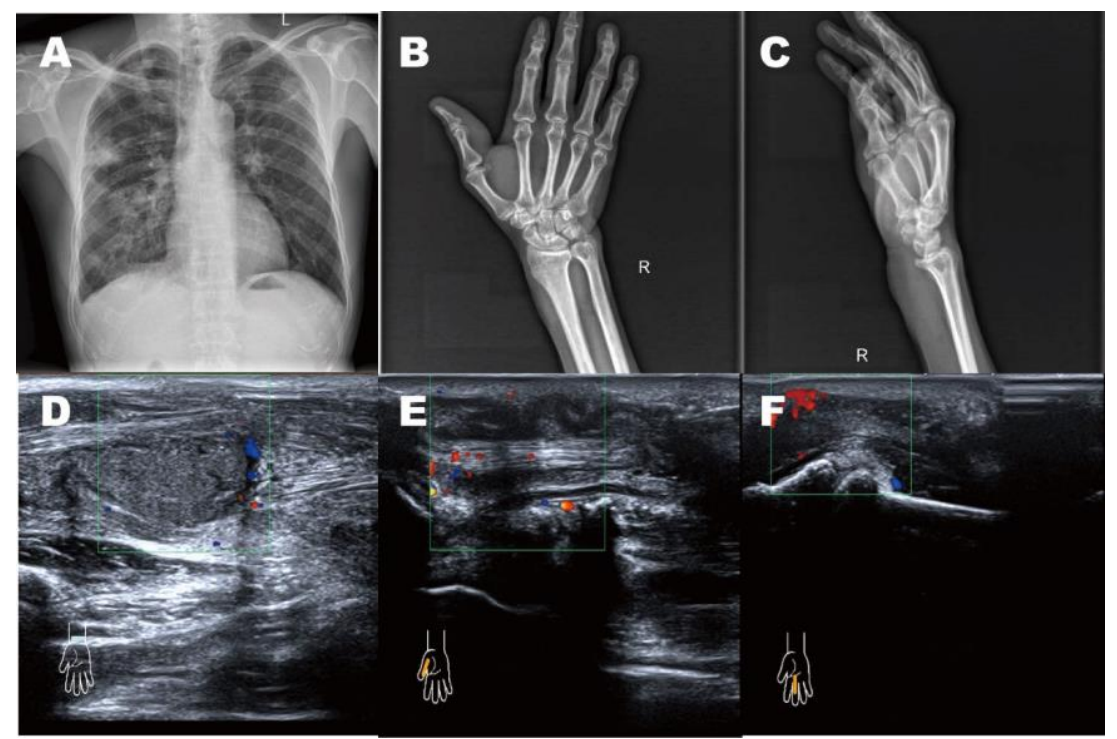

FIGURE 3 (A) Intraoperative appearance of the thumb; (B) Intraoperative appearance of the middle finger; (C), (D) and (E) Intraoperative appearance of the wrist; $(\mathrm{F})$ Total synovectomy done 


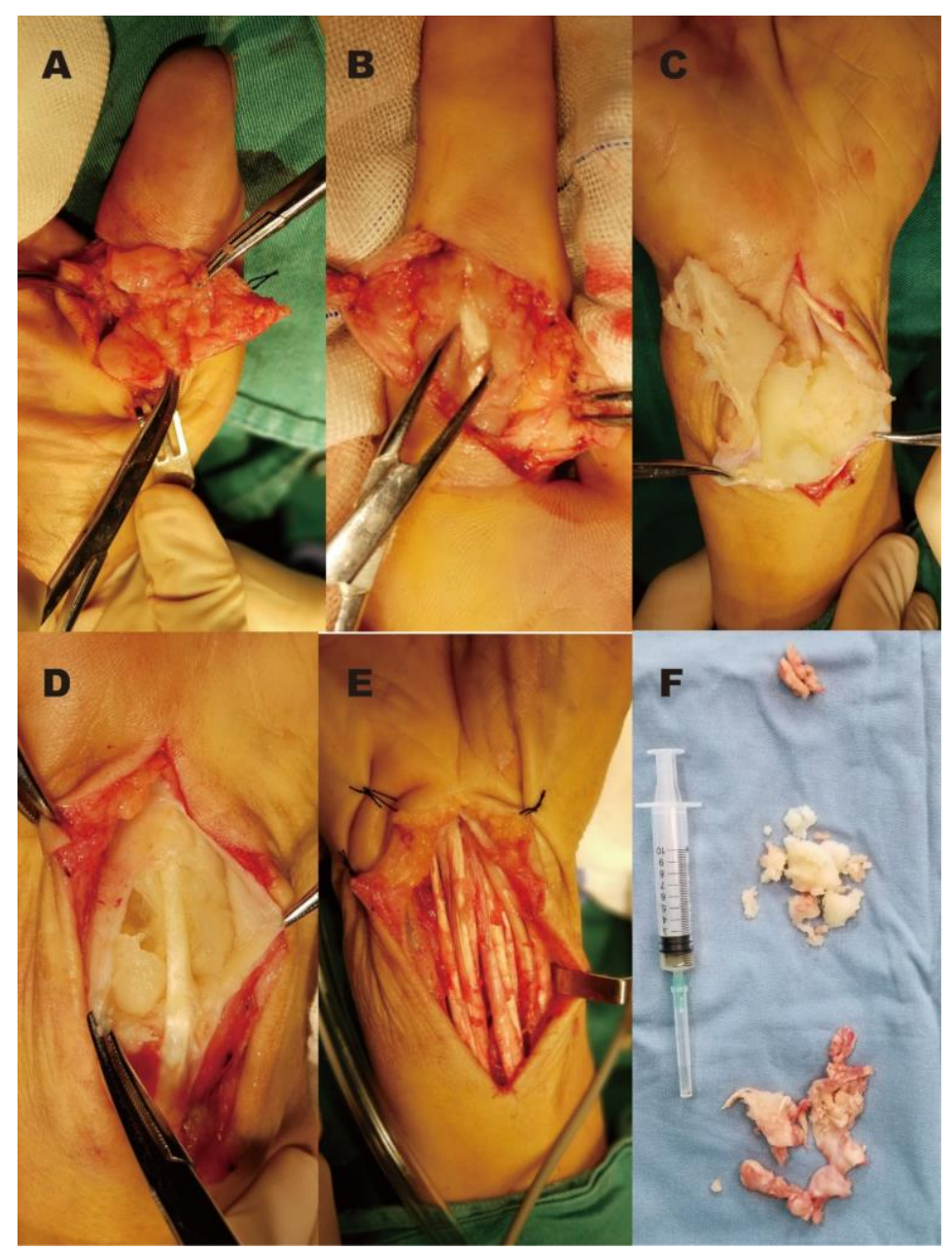

FIGURE 4 (A) and (B) Histopathologic examination showing granulomatous inflammation with Langerhans giant cells; (D) and (E) Two years after surgery and there is no evidence of recurrence

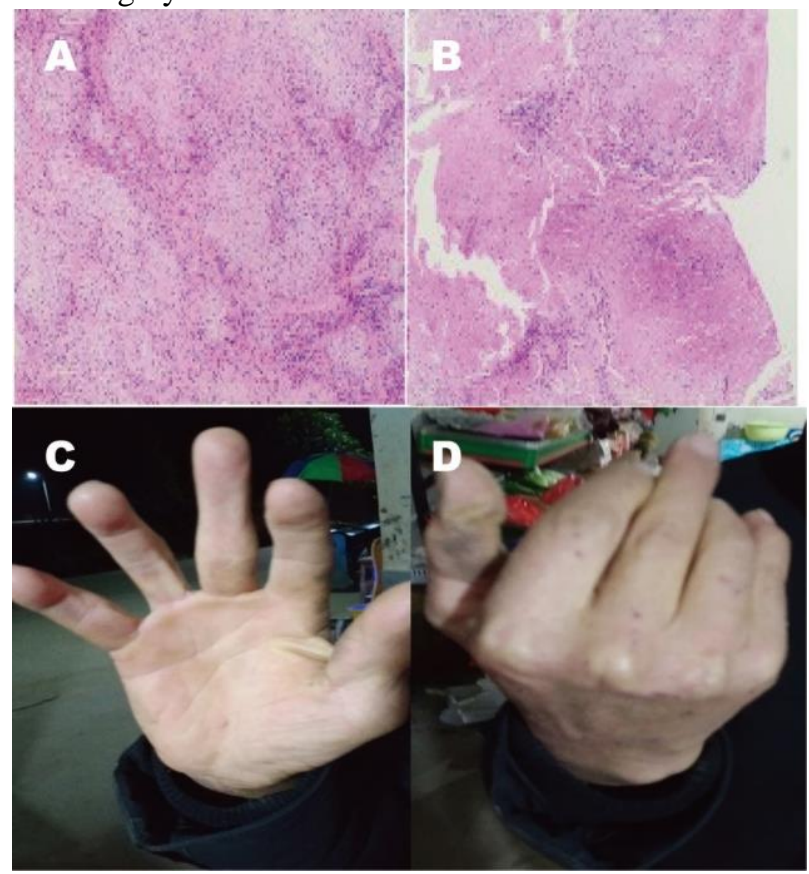




\section{Discussion}

The most common sites of osteoarticular tuberculosis are the spine, hip joint and knee. (5)Hand involvement is rare and occurs only in about $10 \%$ of all bone and joint lesions, mostly on the side of the flexor and the edge of the ulna.(4) Due to the lack of specificity of clinical manifestations, laboratory tests are often negative so that the probability of a missed diagnosis and misdiagnosis is very high. It is generally believed that there are two kinds of pathogenesis of tuberculous tenosynovitis: one is that tuberculosis is caused by hematogenous spread and the other is by direct contact with Mycobacterium tuberculosis.(6) The risk factors for this disease are male, dominant hand, overuse of hand, advanced age, low socio-economic status, malnutrition, extremity trauma and immunosuppression.(7) The main symptoms of the disease are slowly progressive swelling in the early stage and eventually protruding masses of the invaded tendon sheath. $(2,8,9)$ Local masses can be extended by communication between tendon sheaths. When the mass is confined to a tendon sheath, it palpates hard, smooth and inactive. Patient shows no obvious dysfunction so that it is easy to be misdiagnosed as ganglion cyst. When multiple tendon sheaths are involved, the swelling range can invade the whole dorsum of the hand and the palmar side of the wrist, and the mass has a sense of fluctuation and twist pronunciation. Most of the affected area had mild pain and tenderness, the pain aggravated after activity and fatigue, and there were no early symptoms of inflammatory changes except ulceration and mixed infection of the skin. In the late stage, the movement of fingers and wrist is limited, and the symptoms of carpal tunnel syndrome may occur when the lesion oppresses the median nerve. Generally, there are no systemic tuberculosis symptoms. The increase in erythrocyte sedimentation rate and a positive tuberculin test has certain reference significance for diagnosis. In this case, the patient found patchy lungs by chest $\mathrm{x}$-ray examination before operation and the postoperative pathological examination confirmed the diagnosis of tuberculous tenosynovitis. A systematic anti-tuberculosis chemotherapy plan was performed during the perioperative period and there was no recurrence of tuberculosis at the second-year's follow-up.

After the hematogenous dissemination of mycobacterium tuberculosis, it is easy to cause inflammation, hyperemia, edema and exudation of tendon sheath and synovium.(10) In the development of tuberculous tenosynovitis, inflammatory secretions can be changed into serous cellulosic exudates. The lesion starts from the synovium of the tendon sheath and the inflammatory exudate and caseous necrotic lesions gradually enlarge in the synovial cavity to form masses. Those severe lesions can cause tendon adhesion, and even tendon erosion or rupture. $(11,12)$ In this case, multiple masses were found in the right wrist and right hand, but no tendon rupture was found during the operation. However, the postoperative follow-up found that the middle finger mild flexion limitation which indicated that the rehabilitation training has not been strengthened in time.

Tuberculous tenosynovitis is often not diagnosed early because the differentiation from soft tissue tumors may be clinically difficult.(13-16) Recognized risk factors for the development of tuberculous tenosynovitis include middle-aged and elderly manual workers, history of wrist trauma or chronic strain, and history of tuberculosis or history of tuberculosis exposure, conditions more common in the elderly, in whom tuberculous tenosynovitis is most prevalent. Some patients feel mild tenderness while touching the mass and they may complain about pain on the wrist or hand while stretching and flexing fingers.(17) Usually the mass has very limited movement and a carpal tunnel syndrome may occur when the mass is located on the metacarpal side of the carpal. X-ray film shows obvious local soft tissue with occasional bone destruction sign. The erythrocyte sedimentation rate increased. When there are no pulmonary tuberculosis lesions and systemic tuberculosis poisoning symptoms, tuberculin test has a certain significance for the qualitative diagnosis of masses.(18) Ultrasonography is useful for evaluating suspected tenosynovitis while magnetic resonance imaging (MRI) is more sensitive. In our case, the patient is an elderly man with a history of excessive use of hands. He used to be a farmer with low socioeconomic status. Although there was no previous history of tuberculosis or contact, the preoperative X-ray examination found patchy shadows in both lungs and positive tuberculosis antibody test suggested that tuberculous tenosynovitis should be highly suspected.

The principles of tuberculous tenosynovitis treatment include regular systemic anti-tuberculosis treatment in the perioperative period, thoroughly excision of the tendon sheath, synovium, and surrounding affected tissue. $(4,6,19,20)$ During the operation, releasing the tendon adhesion and repair the ruptured tendon is essential. After operation, rehabilitation of the affected wrist and hand should begin as soon as possible. In this case, the patient received anti-tuberculosis treatment for 6 months after operation, and recovered without complications or recurrence of tuberculosis. Therefore, anti-tuberculosis drugs should be taken as soon as possible once the clinical diagnosis is confirmed. The whole treatment plan should be completed to prevent recurrence.

\section{Conclusion}

We described a rare case about a patient with multiple tuberculous tenosynovitis in the right wrist and hand. Through surgical resection and comprehensive anti-tuberculosis chemotherapy plan, the patient was successfully cured and discharged without recurrence. Multiple tenosynovitis should be kept in mind as a rare but possible extrapulmonary tuberculosis presentation 
even though no previous TB history. Laboratory analysis, imaging and microbiology contribute to the diagnosis, but final confirmation usually comes with histopathology. The elementary treatment is anti-tuberculosis drugs and surgery should be performed in cases with wrist and hand pain and other related signs.

\section{Data Availability Statement}

The original contributions presented in the study are included in the article/supplementary materials, further inquiries can be directed to the corresponding author.

\section{Ethics Statement}

Written informed consent was obtained from the individual for the publication of any potentially identifiable images or data included in this article.

\section{Author Contributions}

JL contributed to the writing of the manuscript and designed the figures. DY,XY, YW, and GF collected clinical data and followed the patient during the clinical course. In addition, GF supervised the medical treatment of the patient, reviewed and approved the final version of this article. All authors contributed to the article and approved the submitted version.

\section{Founding}

This work was supported by grants from the Fundamental Research Funds for the Central Universities (21619419) and the Funds for the Central Government Guides the Development of Local Science and Technology (2019SKJ019).

\section{References}

1. Aboudola S, Sienko A, Carey RB, Johnson S. Tuberculous tenosynovitis. Hum Pathol (2004) 35(8):1044-6. doi: 10.1016/j.humpath.2004.04.005. PubMed PMID: WOS:000223440100017.

2. Demir M, Kucuk L, Mermut G, Pullukcu H, Cavusoglu C, Isikgoz Tasbakan M. Tuberculous tenosynovitis in wrist flexor tendons. Tuberk Torak (2019) 67(3):236-8. doi: 10.5578/tt.68451. PubMed PMID: WOS:000493372200013.

3. Bagir M, Sayit E. Tuberculous Tenosynovitis of the Wrist: A Report of Two Cases with Special Reference to Magnetic Resonance Findings. Curr Med Imaging (2019) 15(5):517-20. doi: 10.2174/1573405614666180919141141. PubMed PMID: WOS:000472025700012.

4. Sbai MA, Benzarti S, Boussen M, Maalla R. Tuberculous flexor tenosynovitis of the hand. Int J Mycobact (2015) 4(4):347-9. doi: 10.1016/j.ijmyco.2015.06.003. PubMed PMID: WOS:000372921100015.

5. Sonia R, Boussaid S, Abdelkafi I, Hela S, Ilhem C, Mohamed E. Osteoarticular Tuberculosis: A Retrospective Study of 119 Cases. Ann Rheum Dis (2017) 76:995-. doi: 10.1136/annrheumdis-2017-eular.6911. PubMed PMID: WOS:000413181403044.

6. Brehm TT, Schmiedel S, Addo MM. Tuberculous Tenosynovitis. Dtsch Arztebl Int (2018) 115(42):714-. doi: 10.3238/arztebl.2018.0714a.

PubMed

PMID: WOS:000450975600008.

7. Miladi S, Dhahri R, Hamdi W, Kaffel D, Abid L, Farah F, et al. Wrist synovectomy confirmed tuberculous tenosynovitis in 8 cases: A follow-up study. Egypt Rheumatol (2016) 38(3):257-61 doi: 10.1016/j.ejr.2016.02.004. PubMed PMID WOS:000408670400019.

8. Suwannaphisit S, Ranong NN. Tuberculous tenosynovitis of the Flexor Tendons of the hand and wrist: A case report and mini-review. Ann Med Surg (2020) 57:249-52. doi: 10.1016/j.amsu.2020.07.061. PubMed PMID: WOS:000570132300009.

9. Mansour O, Moussa MK, Khalaf Z, Zreik H, Raad RB Tuberculous Extensor Tenosynovitis Presenting as Huge Mass on the Dorsum of the Hand. Cureus (2020) 12(9). doi: ARTN e10236 10.7759/cureus.10236. PubMed PMID: WOS:000566019300002

10. Jira M, Qacif H, Sekkach Y, El Qatni M, Elouennass M, Ghafir D. Tuberculous tenosynovitis: an uncommon manifestation. Rev Med Interne (2007) 28(1):56-8. doi 10.1016/j.revmed.2006.10.328. PubMed PMID WOS:000243773000014.

11. Jover-Saenz A, Porcel-Perez JM, Madronero-Vuelta AB Gomez-Ribelles A. Tuberculous tenosynovitis in a patient with chronic corticosteroid therapy. Enferm Infec Micr Cl (2005) 23(6):391-2. doi: Doi 10.1157/13076187. PubMed PMID WOS:000230385300016.

12. Emmadi R, Lee J, Teopengko E. Tuberculous tenosynovitis: Identification and speciation from paraffin-embedded tissue. $J$ Mol Diagn (2004) 6(2):152-. PubMed PMID WOS:000221002500021.

13. Chandrasekharan J, Sambandam SN, Cheriyakara S, Mounasamy V. Tuberculous tenosynovitis presenting as finger drop: a case report and a systematic review of the literature Mltj-Muscle Ligament (2016) 6(2):258-63. doi: 10.11138/mltj/2016.6.2.258. PubMed PMID: WOS:000385696400015.

14. Gokkus K, Sagtas E, Aydin AT. Tuberculous Tenosynovitis of the Wrist Mimicking a Volar Wrist Ganglion Cyst and Carpal Tunnel Syndrome. Turk J Rheumatol (2013) 28(2):128-31. doi 10.5606/tjr.2013.2765 PubMed WOS:000320597200010

15. Walker UA, Gutfleisch J, Peter HH. Case number 23 : Tuberculous tenosynovitis. Ann Rheum Dis (2002) 61(5):384-. doi: DOI 10.1136/ard.61.5.384. PubMed PMID: WOS:000175320600002.

16. Esenyel CZ, Bulbul M, Kara AN. Isolated tuberculous tenosynovitis of the flexor tendon of the fourth finger of the hand - Case report. Scand J Plast Recons (2000) 34(3):283-5. PubMed PMID: WOS:000089274100017.

17. Karunadasa KP, Dissanayake DA, Beneragama TS, Perera D. Staged flexor tendon reconstruction in a patient with caseous tuberculous tenosynovitis. J Hand Surg-Eur Vol (2010) 35e(6):515-6. doi: 10.1177/1753193409360604. PubMed PMID WOS:000279760600019.

18. Gupta L, Gupta V, Kumar T. Rice Bodies in Tuberculous Tenosynovitis of Wrist. Reumatol Clin (2018) 14(5):314-6. doi 10.1016/j.reuma.2017.08.003. PubMed PMID: WOS:000444033400014.

19. Samson M, Roch N, Audia S, Berthier S, Leguy V, Bonnotte B, et al. Tuberculous tenosynovitis. Presse Med (2011) 40(9):877-81. doi: 10.1016/j.lpm.2011.02.032. PubMed PMID WOS:000295384800012.

20. Amine B, Benbouazza K, Bahiri R, Hajjaj-Hassouni N. Multifocal tuberculous tenosynovitis. Joint Bone Spine (2006) 73(4):474-5. doi: 10.1016/j.jbspin.2005.08.002. PubMed PMID WOS:000239745500026. 